\title{
Le Bulletin change de nom et de formule
}

\author{
Le Comité de rédaction : · J.-P. Chippaux · A. Epelboin · P. Gazin · J. F. Pays · É. Pichard
}

Reçu le 18 janvier 2021; accepté le 2 février 2021

(C) Société de pathologie exotique et Lavoisier SAS 2021

Fondé en 1908 par Alphonse Laveran (prix Nobel de physiologie ou de médecine 1907), tout d'abord au service de la médecine coloniale puis des médecines nationales et internationales, le Bulletin de la Société de pathologie exotique est une référence internationale dans la recherche et la réflexion sur les maladies et la santé publique tropicales depuis plus d'un siècle.

La communauté francophone mondiale des praticiens et des chercheurs, biologistes, médecins, vétérinaires, pharmaciens et spécialistes de sciences humaines qui travaillent sur les maladies tropicales s'est aujourd'hui considérablement diversifiée et élargie, notamment dans les pays tropicaux, de même que l'approche des problèmes sanitaires et de leur prise en charge. Plus large, confrontée à des stratégies préventives ou thérapeutiques et des problématiques de développement plus complexes, cette communauté manque d'accès aux publications de travaux scientifiques en langue française et, de ce fait, est souvent écartée des débats médicaux, scientifiques et sociétaux.

Aujourd'hui, le Bulletin se transforme pour mieux servir l'approche intégrée, systémique et unifiée de la santé individuelle, publique, animale et environnementale dans les pays tropicaux : il devient entièrement numérique, sans support papier autre que les impressions réalisées individuellement. Il offrira une réponse cohérente et ouverte à la demande croissante de publications en ligne, rapides et libres d'accès. Refusant le principe de frais de publication à la charge des auteurs, il abandonne l'abonnement payant à la revue et opte pour un accès libre et gratuit.

Ce numéro est donc le dernier imprimé et distribué par voie postale.

La Société francophone de médecine tropicale et santé internationale (SFMTSI), nouveau nom de la Société de pathologie exotique (SPE), a fait le choix de remplacer le

Le Comité de rédaction : - J.-P. Chippaux · A. Epelboin .

P. Gazin · J. F. Pays · É. Pichard

Société francophone de médecine tropicale et santé internationale (ancienne SPE)

Hôpital Pitié-Salpêtrière - Pavillon Laveran,

47-83 Bld de l'Hôpital

F-75651 Paris cedex 13, France

revue@societe-mtsi.fr
Bulletin de la SPE par une nouvelle revue appelée Médecine tropicale et santé internationale (MTSI), toujours référencée sur les principaux moteurs de recherche d'articles scientifiques et génériques. Il fait suite au Bulletin de la Société de pathologie exotique et à Médecine et santé tropicales (ex-Médecine tropicale).

MTSI sera composé de deux sections, le Bulletin et le Magazine, outre l'accès gratuit aux fonds documentaires (Bulletin de la Société de pathologie exotique 1908-2020, photos et vidéos, bibliothèque...).

Le nouveau Bulletin est dirigé par le même comité de rédaction et un conseil scientifique élargi, notamment à l'international (https://societe-mtsi.fr/equipe/), dont les membres sont choisis en fonction de leurs compétences et de leur participation active à la lecture et à l'accompagnement des articles. Il continuera de publier des articles scientifiques soumis à des relecteurs compétents et didactiques, avec le même niveau d'exigence que celui du Bulletin de la Société de pathologie exotique. Il poursuivra ses objectifs scientifiques et pédagogiques en accompagnant la publication, voire la rédaction de travaux de chercheurs ou de cliniciens peu au fait des normes d'une publication scientifique de niveau international.

En dehors de numéros spéciaux, les articles seront publiés au fur et à mesure de leur acceptation par le comité scientifique selon les mêmes rubriques que précédemment, susceptibles d'évolution: Anthropologie médicale ; Bactériologie ; Biologie clinique ; Chirurgie ; Clinique ; Économie de la santé ; Entomologie médicale ; Envenimations ; Épidémiologie ; Histoire de la médecine ; Lutte antivectorielle ; Médecine vétérinaire ; Mycologie ; Parasitologie ; Santé publique ; Statistiques hospitalières ; Thérapeutique ; Toxicologie ; Toxinologie ; Virologie.

Ouvert à l'ensemble des personnels de santé humaine et animale, dans l'esprit pluridisciplinaire d' «Une seule santé » (one health), il publiera des revues thématiques, des travaux originaux, des cas cliniques, de courtes notes, des comptes rendus de congrès, des résumés de communications orales ou affichées, diaporamas des présentations.

Dépassant le cadre des pathologies tropicales, le Bulletin s'intéressera à toutes les pathologies ou questions de santé, 
quelle que soit la discipline, dès lors que l'exercice et le contexte tropical dans lesquels elles seront décrites seront mis en évidence et développés. Prenant en compte la transition épidémiologique, le nouveau Bulletin s'intéressera aux pathologies cosmopolites, notamment chroniques, se développant dans les pays tropicaux ou dans les diasporas de ces pays, lorsqu'elles seront présentées dans le contexte des systèmes de santé locaux ou feront référence à l'adaptation nécessaire à leur prise en charge, en mettant l'accent sur les particularités logistiques, écologiques, culturelles, socioéconomiques et politiques de l'offre de soins. Une attention toute particulière continuera d'être accordée à des publications concernant des sites suivis depuis des décennies.

Le Magazine, nouvelle section de MTSI, dirigé par un comité de rédaction différent de celui du Bulletin, rendra compte de la santé tropicale dans le contexte «Une seule santé », au travers de sujets d'actualité en santé tropicale, en dehors du cadre formel des articles originaux de revues scientifiques (http://revuemtsi.societe-mtsi.fr/index.php/bspe-mag).

Il publiera des questions d'actualité de la santé tropicale, des fiches techniques de terrain, des présentations d'innovations ou de projets, des débats, des éclairages, des comptes rendus de réunions, des focus sur la situation d'un pays ou d'une région et de nombreuses rubriques didactiques (Synthèse ; Quel est votre diagnostic ? PharmaTrop ; VeninTrop ; HistoTrop, etc.) et des tribunes ou prises de position face aux problématiques de la santé au Sud.

Les articles de MTSI, gratuits pour les auteurs comme pour les lecteurs, seront désormais accessibles sur le site www.revuemtsi.societe-mtsi.fr. 